\section{IDSORT: An individual differences multidimensional scaling program for sorting data}

\section{YOSHIO TAKANE \\ McGill University, Montreal, Quebec H3A 1B1, Canada}

IDSORT is an individual differences multidimensional scaling (MDS) program specifically designed for sorting data. It is a three-way extension of the MDSORT program (Takane, 1980, 1981). While the MDSORT program finds a single stimulus configuration from $\mathrm{N}$ sets of sorting data, IDSORT also obtains dimensional weights for subjects, which account for certain individual differences in sorting behavior. That is, IDSORT fits the INDSCAL (Carroll \& Chang, 1970) type of the weighted euclidean distance model to sorting data.

The stimulus sorting method, in which the subjects are asked to sort a set of stimuli into several groups (clusters) in terms of their similarity, has been very popular among social scientists because of its simplicity. Yet when the sorting data are analyzed by a nonmetric MDS procedure, such as ALSCAL (Takane, Young, \& de Leeuw, 1977), there is some uncertainty as to how similarity measures should be defined based on the sorting information. IDSORT, on the other hand, simultaneously scales the sorting data (i.e., it derives a similarity measure from the sorting data) and represents them (i.e., it finds a stimulus representation from the derived similarity measure) based on a single common criterion. IDSORT is the first individual differences MDS method that directly applies to the sorting data.

Description. IDSORT allows a user to directly input original sorting data. The program then derives similarity matrices (one for each subject) between the stimuli and finds a stimulus configuration and dimensional weights for the subjects. The stimulus configuration and the dimensional weights are determined in such a way that the sum of squared intercluster distances in the subject space (summed over the subjects) is maximized under suitable normalization restrictions. As in the MDSORT program, cluster centroids for the stimuli classified into certain clusters by each subject may optionally be calculated. These provide information concerning another source of individual differences in sorting behavior. Unlike two-way MDS, lower dimensional solutions are not proper subsets of a higher dimensional solution. So, separate analyses have to be conducted to obtain solutions with different dimensionalities.

The preparation of this manuscript was supported by Grant A6394 to the author from the Natural Sciences and Engineering Research Council of Canada.
Input and Output. Input to the program is rather similar to that of MDSORT. It consists of several job parameters (title, number of stimuli, subjects, and dimensions, input data format, and input/output options) and the data for $\mathrm{N}$ subjects. Stimulus labels and subject labels may optionally be input. All information should be punched on IBM cards in a prescribed format. The sorting data are indicated by numbers indicating cluster memberships of stimuli. For example, "1 22313 " on a data card implies that Stimuli 1 and 4 are put into a same cluster, Stimuli 3 and 5 into another cluster, and Stimulus 2 into a cluster of its own. Missing data may be handled by assigning additional cluster numbers.

The program prints out stimulus coordinates and individual differences weights for a specified dimensionality. These may optionally be plotted and/or punched on cards. Cluster centroids may also be calculated for each subject, printed, plotted, and/or punched out.

Language and Computer. IDSORT is written in FORTRAN IV for use on a batch processing system with an ordinary line printer.

Availability. A copy of the program and the program user's manual (Takane, Note 1) can be obtained from Yoshio Takane, Department of Psychology, McGill University, 1205 Avenue Docteur Penfield, Montreal, Quebec H3A 1B1, Canada. Requests should be accompanied by a $\$ 20$ check (made payable to McGill University) to cover necessary costs, which include a new ninetrack magnetic tape. Do not send cash or a tape. The MDSORT (Takane, 1981) program can be written on the same tape, if requested.

\section{REFERENCE NOTE}

1. Takane, Y. How to use IDSORT: An individual-differences multidimensional scaling for sorting data. Unpublished user's manual, McOill University, 1982.

\section{REFERENCES}

Carroll, J. D., \& Chano, J. J. Analysis of individual differences in multidimensional scaling via an $\mathbf{N}$-way generalization of "Eckart-Young" decomposition. Psychometrika, 1970, 35, 283-319.

Takane, Y. Analysis of categorizing behavior by a quantification method. Behaviormetrika, 1980, 8, 75-86.

TAKANE, Y. MDSORT: A special-purpose multidimensional scaling program for sorting data. Behavior Research Methods \& Instrumentation, 1981, 13, 698.

Takane, Y., Youna, F. W., \& DE LeEuW, J. Nonmetric individual differences multidimensional scaling: An alternating least squares method with optimal scaling features. Psychometrika, $1977,42,7-67$.

(Accepted for publication October 12, 1982.) 